1 KNOWLEDGE DESIRED BY EMS MANAGERS OF THEIR AMBULANCE CLINICIANS

1,2 M Holmberg*, ${ }^{3} \mid$ Fagerberg, ${ }^{4} \mathrm{AC}$ Wahlberg. 'School of Health, Care and Social Welfare, Mälardalen University, Eskilstuna, Sweden; ${ }^{2}$ Ambulance Service Department, Sörmland County Council, Eskilstuna, Sweden; ${ }^{3}$ Department of Health Care Sciences, Ersta Sköndal Bräcke University College, Stockholm, Sweden; ${ }^{4}$ Department of Neurobiology, Care Sciences and Society, Karolinska Institute, Stockholm, Sweden

\subsection{6/10.1136/bmjopen-2018-EMS.1}

Aim Emergency medical service (EMS) managers are responsible for organisational tasking. In this they are dependent on the knowledge possessed by their ambulance clinicians (ACs). Knowledge in EMS may be described as embracing formal education, protocol dependent and containing tacit experienced-based dimensions. However little is known about EMS managers' approach to this knowledge. The aim of the study was therefore to identify the types of knowledge that Swedish EMS managers considered desirable in their ACs.

Methods Thirty-six EMS managers participated in a modified Delphi study. Twenty-six sub-categories emerged in the first openended questionnaire. Following the EMS managers were encouraged to rate each sub-category, and the ten with the highest mean were interdependently ranked in the final round.

Results The sub-categories covered knowledge related to; contextual aspects, medical and holistic assessments, formal education and organisational issues. Eventually, the sub-category 'Knowledge to assess the patient's situation from a holistic perspective' was the highest ranked, followed by 'Medical knowledge to assess and care for different diseases' and 'Knowledge to be able to care for critically ill patients'.

Conclusion The EMS organisations' primary focus is on providing emergency medical care and treatment. Hence, the present result may have impact on this in order to cover more complex types of knowledge, addressing essentially medical care, contextual aspects and nursing. The boundaries between these may sometimes be elusive, challenging the ACs to balance these areas of knowledge in a complex context.

Conflict of interest We declare no conflict of interest.

Funding This study was funded by $\mathrm{R}$ and D Centre, Sörmlands County Council.

\section{EARLY MULTIDISCIPLINAIRY SCREENING OF DYSPHAGIA AT ADMISSION TO THE EMERGENCY DEPARTMENT - A PILOT STUDY}

${ }^{1}$ LR Sørensen*, ${ }^{2} D$ Sandager, ${ }^{1} \mathrm{~A}$ Jørgensen, ${ }^{2} \mathrm{AB}$ Christensen, ${ }^{1} \mathrm{M}$ Ludwig, ${ }^{3} \mathrm{P}$ Leutscher, ${ }^{2,3}$ D Melgaard. 'Department of Emergency Medicine, North Denmark Regional Hospital, Denmark; ${ }^{2}$ Physio- and Occupational Therapy Department, North Denmark Regional Hospital, Denmark; ${ }^{3}$ Center for Clinical Research, North Denmark Regional Hospitall Department of Clinical Medicine, Aalborg University, Denmark

\subsection{6/10.1136/bmjopen-2018-EMS.2}

Aim Knowledge about effect of early dysphagia screening is limited. The aim of this study is to examine the prevalence of dysphagia in the Emergency Department (ED) population.

Method This study included consecutively hospitalised patients in 10 days from 2 pm-10 pm at the ED of North Denmark Regional Hospital. The screening took place within 2 hours of admission. Inclusion criteria were any of the following: age $\geq 65$ years, neurological disorders, alcoholism, COPD, pneumonia, dyspnoea, diabetes or unexplained weight loss. A nurse screened patients with a water test and with signs of dysphagia tested by an occupational therapist with the V-VST and the MEOF-II.
Results Of 140 eligible patients (56\% male, median age 75 years) $95(68 \%)$ were screened. It was impossible to screen 12 patients (9\%) to limited time and 30 patients (21\%) due to poor health condition and 5 patients (4\%) declined participation. The prevalence of dysphagia in the study population was $16 \%$ (15 patients). Results from the water test were confirmed with V-VST and MEOF-II. In patients with lung related diseases or circulatory diseases was the prevalence respectively $25 \%$ and $24 \%$. Patients, not screened due to poor health condition, were tested during hospitalisation and the prevalence of dysphagia was $75 \%$ in this group of patients.

Conclusion The prevalence in ED patients was $16 \%$. Patients transferred to other departments due to poor health condition had prevalence of $75 \%$. It is possible to screen patients in the ED. The water test is a useful screening tool in an acute setting.

Conflict of interest No conflicts of interest

Funding No funding

\section{AN EXPLORATION OF THE EFFECTS THAT FREQUENT EXPOSURE TO LIFE-THREATENING EVENTS MAY HAVE ON A CRITICAL CARE PARAMEDIC'S PSYCHOLOGICAL WELLBEING?}

E Vyvyan*. University of Surrey, Faculty of Health and Medical Sciences, Stag Hill, Guildford, Surrey

\subsection{6/10.1136/bmjopen-2018-EMS.3}

Aim UK Critical Care Paramedic (CCPs) are a specialty of experienced paramedics who have received higher education to deliver extended clinical care. Due to their specialist skills they are dispatched to the most critically ill and injured patients to deliver advanced care. Internationally, CCPs are recognised within the USA and Canada as Advanced Care Paramedics and as Mobile Intensive Care Ambulance Paramedic (MICA) in Australia.

Method The CCPs studied within this UK Ambulance Trust have all received post graduate training achieving a certificate, diploma or MSc in Advanced Paramedic Practice or Critical Care. Eight qualified CCPs with at least one years' experience as a CCP, were recruited from one UK Ambulance Service. Face to face, semi structured interviews were conducted. Descriptive phenomenology and Colaizzi's framework for data analysis was used to discover the personal perspectives and experiences of CCPs.

Results CCPs see a range of highly emotive incidents on a daily basis, specifically cardiac arrests and traumatically injured patients. They identified with symptoms suggestive of a heightened emotional response. The negativity of trauma identified within the studies literature review was not comparable with the responses from the majority of CCPs. The CCPs demonstrate resilience and adopt a diverse range of conscious coping strategies. The following factors were appraised by the CCPs to enhance their coping strategies: their ability to perform advanced clinical interventions and perceived survival of patients. Organised debriefs and improved clinical reflection. Enhanced inter-professional relationships and a regular crewmate for support.

Conclusion CCPs felt the role caused slight disruption to their health and wellbeing. They implied improvements to the ambulance services' counselling. Further research focussing on larger populations adopting CCP and advanced paramedic groups would be recommended.

Conflict of interest None

Funding None 